\title{
Priority Polling with Reservation Wireless Access Protocol for Multimedia Ad Hoc Networks*
}

\author{
Chih-Cheng Tseng \\ cctseng@mail.jwit.edu.tw \\ Kwang-Cheng Chen \\ chenkc@cc.ee.ntu.edu.tw \\ Graduate Institute of Communication Engineering, National Taiwan University, Taipei, Taiwan, R.O.C.
}

\begin{abstract}
In this paper, a new traffic source, Priority Data, is considered to access the channel with the highest priority among conventional CBR, VBR and ABR sources. In order to achieve and maintain $Q 0 S$ guarantee for admitted $C B R$ and VBR sources, a QoS guaranteed wireless access protocol, the Priority Polling with Reservation (PPR) protocol that consists of Priority Polling Scheme (PPS) and Randomly Addressed Polling (RAP) is proposed. We provided the jitter and delay constraints for CBR and VBR sources by reserving bandwidth for the mean PD sources that active during the time to transmit CBR and VBR sources. The results show that under the preemptive of $\mathrm{PD}$ sources, the QoS constraints for CBR and VBR sources are guaranteed.
\end{abstract}

\section{INTRODUCTION}

In the recent years, the topic of wireless ad hoc networks has received increasing attention [1]-[3]. A wireless ad hoc network is a mobile, multihop wireless network without existence of fixed infrastructures. Most previous works, e.g. [4]-[7], classified the multimedia traffic into CBR, VBR, and $A B R$. In this paper, we further to investigate a new traffic source, Priority Data (PD), which is sensitive to both delay and error and is with the highest priority to access the channel whenever it is generated. Application of the PD traffic sources can be found both in the military and commercial. In modern multimedia military applications, the encrypted or scrambled emergency data or tactical commands need to be transmitted immediately in spite of the scheduled CBR and VBR sources that have been admitted. In the commercial applications, some critical control signals such as handoff in the wireless mobile cellular communications and topology information in the wireless ad hoc networks all need to be transmitted with the highest priority in order to maintain the continuity of the ongoing communications or the robustness of the networks. Since the irregularity and burstiness of the PD traffic sources and the highest channel access priority, the QoS provisions, jitter and delay, of the admitted CBR and VBR sources need to be handled much more carefully. Thus, our objectives are to design a wireless access protocol to:

1. Allow PD sources to access the channel with the highest priority under the scheduled polling based protocol.

2. Minimize the impact of PD traffic sources on the admitted CBR and VBR traffic sources.

3. Maximize the number of CBR and VBR traffic sources that can be admitted.

The rest of the paper is organized as follows. In Section II,

*This research is supported in part by the Chung-Shan Institute of Science and Technology and by Excellent Project, Taiwan, R. O. C. under Contract $88 C S D 002002$ and 89EFA06247. we propose the Priority Polling with Reservation (PPR) wireless access protocol. In Section III, we derived the jitter and delay bounds for CBR and VBR traffic sources respectively. The simulation results and discussions are presented in Section IV, and Section V concludes this paper.

\section{PRIORITY POLLING with RESERVATIOIN PROTOCOL}

\section{A. Assumptions}

We assumed the entire network is partitioned into a set of clusters [4][5][8]. Every cluster has its own clusterhead $(\mathrm{CH})$ that acts as a local controller for the nodes in that cluster. All nodes belong to the same cluster are in direct communication range of the $\mathrm{CH}$ and any transmission must send to the $\mathrm{CH}$ first. The channel is assumed to be noise-free and all packets with the same size. The characteristics of the traffic sources we considered are stated below.

- A PD traffic source is a Poisson process with mean arrival rate $\lambda_{P D}$ and with the highest priority to access the channel.

- A CBR source $i$ is modeled by $\left(r_{c, i}, j_{i}, l_{i}\right)$, where $r_{c, i}$ is the average rate, $j_{i}$ is the maximum tolerable jitter and $l_{i}$ is the average tolerable packet loss rate.

- A VBR source $i$ is modeled by $\left(r_{v, i}, b_{i}, d_{i}\right)$, where $r_{v, i}$ is the average rate, $b_{i}$ is the maximum burstiness, and $d_{i}$ is the maximum tolerable delay. Besides, we regulate VBR source $i$ by a $\left(b_{i}, r_{v, i}\right)$-leaky bucket. Packet passing through the leaky bucket is stored in RTT buffer.

- An ABR source is considered as a Poisson process with mean arrival rate $\lambda_{A B R}$. The ABR traffic sources have neither jitter constraint nor delay constraint. ABR sources share fairly and efficiently the remaining bandwidth from the PD, CBR and VBR sources.

\section{B. The PPR protocol}

In order to achieve the objectives mentioned in Section I, we proposed the Priority Polling with Reservation (PPR) wireless access protocol, which consists of Priority Polling Scheme (PPS) and Randomly Addressed Polling (RAP) [9]. The system operated as follows: When there is no active PD source, the system follows the operation of the PPS. Once PD traffic source is generated, the system switches to the RAP protocol until all pending PD packets are transmitted. During the period to transmit the PD packets, all scheduled CBR and VBR transmissions are deferred. If the deferred time of a scheduled CBR/VBR packet violated its jitter/delay provision, 
it is discarded and removed from the corresponding Ready-To-Transmit (RTT) buffer. The PPR model is shown in Figure 1.

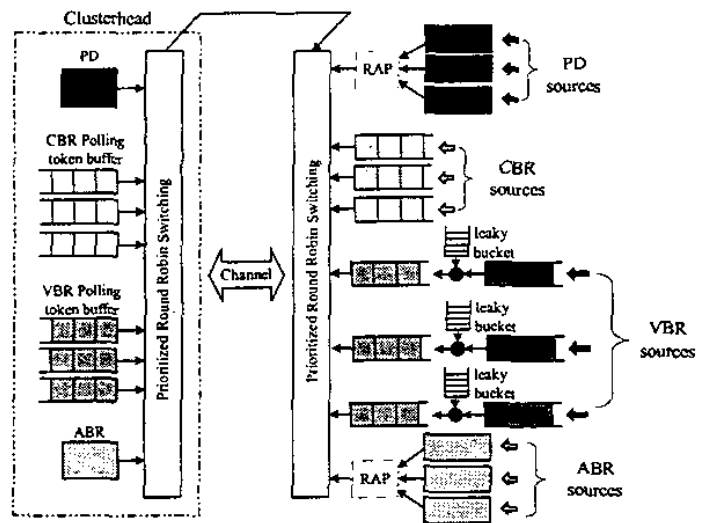

Figure 1 Service Model of the Priority Polling with Reservation Method.

\begin{tabular}{|c|c|c|c|c|c|c|c|c|c|c|}
\hline 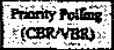 & pots & n n & poll & 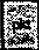 & $\mid c x+p d$ & 6 & sck & 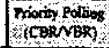 & $\begin{array}{l}\text { RAP } \\
\text { (ABR) }\end{array}$ & $\begin{array}{l}\text { Priony Aollos } \\
\text { (CERNBR) }\end{array}$ \\
\hline
\end{tabular}

Figure 2 An example access timing of the PPR.

\section{Priority Polling Scheme (PPS)}

1. For each admitted $\left(r_{c, i}, j_{i}, l_{i}\right)$ CBR source with, its polling token is generated every $1 / r_{c, i}$ second in the $\mathrm{CH}$.

2. For each admitted $\left(r_{v, i}, b_{i}, d_{i}\right)$ VBR source with, its first polling token is generated in the $\mathrm{CH} p_{i}$ second after the connection is set up, where $p_{i}$ is a design parameter to meet its delay constraint.

3. When the channel is idle (no transmission or at the end of each CBR/VBR/ABR transmission),

3.1 The $\mathrm{CH}$ first polls the PD sources. If there is active PD source, the $\mathrm{CH}$ services the PD source based on the RAP protocol until the PD source are depleted.

3.2 If there is no active PD source, the $\mathrm{CH}$ scans the CBR polling token buffer according to the increasing priority order. If polling token buffer is non-empty, it poils the corresponding CBR source to send a packet from RTT buffer and then removes the polling token.

3.3 If no active PD source and all CBR polling token buffers are empty, the $\mathrm{CH}$ switches to scan the VBR polling token buffers according to the increasing order. If polling token is found, the $\mathrm{CH}$ polls the corresponding VBR source without removing the polling token.

3.3.1 After receiving the poll signal, the VBR source sends out a packet if there is one in its RTT buffer; otherwise, it sends an End-Of-File (EOF) signal.

3.3.2 When an EOF signal from a VBR source is received, the $\mathrm{CH}$ removes the polling token from the polling token buffer of that source and schedules the generation of the next polling token after $p$ seconds.

3.4 When no active PD, CBR and VBR sources are detected, the ABR sources are serviced one packet at a time based on the RAP protocol.

\section{Randomly Address Polling (RAP)}

Since the randomness of $\mathrm{PD}$ and ABR sources, a random access protocol is needed to efficiently and fairly utilize the available bandwidth. Here, due to better performance, we select the RAP protocol to govern transmission of PD and ABR packets. The operation of RAP is briefly stated as follows.

1. At the beginning of every RAP cycle, the $\mathrm{CH}$ broadcasts a POLL signal to invite transmission.

2. After receiving the POLL signal, every active PD or ABR source simultaneously transmits an "address" which is randomly selected from the set $C_{R}$ to the $\mathrm{CH}$. If there is no address detected, terminates this procedure.

3. The $\mathrm{CH}$ polls active sources with the marked addresses one by one to transmit packet.

4. The $\mathrm{CH}$ will successfully receive a packet if there is only one active source selects the polled address. Otherwise, packet collision occurs.

Figure 2 is an example access timing of the PPR protocol. In Figure 2, after polling some CBR and VBR sources, PD sources access the channel through the RAP protocol. After depleting the PD sources, the $\mathrm{CH}$ continues the priority polling. When all higher priority sources are depleted, the active ABR sources access the channel until higher priority sources are generated again.

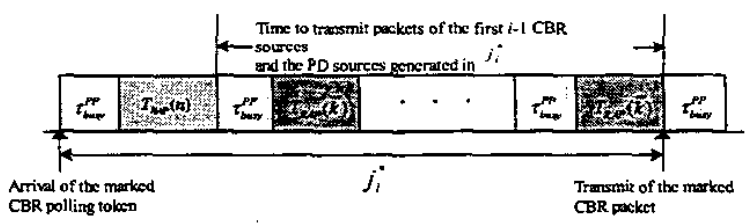

Figure 3 Computation of the jitter of the $i$-th CBR source.

\section{Qos CONSTRAINT ANALYSIS}

In the section, we provide the jitter and delay constraints for CBR or VBR source in the system. Let $\tau_{\text {poll }}, \tau_{\text {data }}$, and $\tau_{\text {mark }}$ be the time to poll a source, to send a packet and to transmit and detect the random address. Let the total time required to transmit a packet under the operation of PPS be $\tau_{\text {busy }}^{P P}=\tau_{\text {poll }}+\tau_{\text {data }}$. Assume there are totally $N$ nodes within the considered cluster and among which there are $n_{c}$ active CBR sources, $n_{v}$ active VBR sources. To study the impact of the PD sources on the QoS provisions of guaranteed CBR and VBR sources, we assume the remaining $n=N-n_{c}-n_{v}$ nodes are all PD sources. The total arrival rate for the $n$ PD sources is $\lambda=\sum_{i=1}^{n} \lambda_{P D}$ for assuming that each PD source with the same arrival rate. 


\section{A. Jitter Analysis for CBR sources}

Theorem 1: Let $\bar{k}$ be the average number of $\mathrm{PD}$ packets that generated during the polling period $\tau_{\text {busy }}^{P P}$. The jitter bound suffered for the $i$-th CBR source, $j_{i}^{*}, i=1,2, \ldots, n_{c}$, is

$$
j_{i} \leq \frac{i \tau_{b u s y}^{P P}+(i-1) \bar{T}_{R A P}(\bar{k})+\bar{T}_{R A P}(n)}{1-\sum_{m=1}^{i-1} r_{c, m}\left[\tau_{b u s y}^{P P}+\bar{T}_{R A P}(\bar{k})\right]},
$$

where $\bar{T}_{R A P}(n)$ (See Appendix) is the average busy period for the RAP protocol to service $n$ active PD sources.

Proof: Assume that a marked polling token of the $i$-th CBR source is generated at time 0 . Consider the case shown in Figure 3, on arriving of the marked polling token, the $\mathrm{CH}$ is transmitting a packet. In this case, the jitter of the marked polling token must to wait $\tau_{b u s y}^{P P}$ for this packet to finish transmission. During this waiting period, we assume all $n$ PD sources are all active. The average time to transmit $n$ active PD sources is $\bar{T}_{R A P}(n)$. Let the jitter bound of the $i$-th CBR polling token be $\dot{j_{i}^{*}}$. According to service priority, the number of polling tokens for the first $i-1$ CBR sources generated in $\dot{j}_{i}^{*}$ can be bounded by $\sum_{m=1}^{i-1}\left[r_{c, m} \dot{j}_{i}^{*}\right]$. Since the number of PD packets generated in the period of $\tau_{\text {busy }}^{P P}$ is a binomial distribution with parameters $n$ and $\left(1-e^{-\lambda_{p D} t_{b e s}^{p p}}\right)$, the average number of active PD packets, $\bar{k}$, is $\bar{k}=\left[n\left(1-e^{-\lambda P D x_{\text {mas }}^{p p}}\right)\right]$. Therefore, the jitter bound for this marked polling token is $\dot{j}_{i}^{*} \leq \tau_{\text {busy }}^{P P}+\bar{T}_{R A P}(n)+\sum_{m=1}^{i-1}\left[r_{c, m} \dot{j}_{i}^{*}\right]\left(\bar{T}_{R A P}(\bar{k})+\tau_{\text {busy }}^{P P}\right)$. Using the fact $\lceil x\rceil \leq x+1$ and after some manipulations, we have

$$
j_{i}^{*} \leq \frac{i \tau_{b u s y}^{P P}+(i-1) \bar{T}_{R A P}(\bar{k})+\bar{T}_{R A P}(n)}{1-\sum_{m=1}^{i=1} r_{c, m}\left[\tau_{b u s y}^{P P}+\bar{T}_{R A P}(\bar{k})\right]} .
$$

QED-

\section{B. Delay Analysis for VBR sources}

Theorem 2: If $\sum_{m=0}^{n_{v}} X_{m}<1$, the delay bound of the $i$-th VBR source, $d_{i}^{*}, i=1, \ldots, n_{v}$ is

$$
d_{i}^{*}=p_{i}+\frac{\sum_{m=0}^{i} Y_{m}+\sum_{m=1}^{i-1} d_{m}^{*} r_{v, m}\left(\tau_{b u s y}^{P P}+\bar{T}_{R A P}(\bar{k})\right)+(i-1) \tau_{\text {empry }}}{1-\sum_{m=0}^{i-1} X_{m}}(2)
$$

where $\quad X_{0}=\sum_{i=1}^{n_{c}} r_{c, i}\left(\tau_{\text {busy }}^{P P}+\bar{T}_{R A P}(\bar{k})\right), \quad Y_{0}=\sum_{i=0}^{n_{\mathrm{c}}} \tau_{\text {busy }}^{P P}+$ $\sum_{i=1}^{n_{c}} \bar{T}_{R A P}(\bar{k})+\bar{T}_{R A P}(n) \quad, \quad X_{m}=r_{v, m}\left(\tau_{b u s y}^{P P}+\bar{T}_{R A P}(\bar{k})\right)+\frac{\tau_{\text {mop } n}}{p_{m}}$ and $Y_{m}=\left(b_{m}+1\right)\left(\tau_{b u s y}^{P P}+\bar{T}_{R A P}(\bar{k})\right)$ for $m=1,2, \ldots, i-1$. QED=

Proof: First, we consider the delay of the first VBR source. According to the preset service discipline in the PPS, the
CBR sources have higher priority to be serviced than VBR sources. The total numbers of $C B R$ packets generated at time $\left(t_{1}, t_{2}\right)$ are bounded by $N_{c}\left(t_{1}, t_{2}\right) \leq \sum_{i=1}^{n_{c}}\left[r_{c, i}\left(t_{2}-t_{1}\right)\right]$. The available service time for the first active VBR source during $\left(t_{1}, t_{2}\right), A_{v, 1}\left(t_{1}, t_{2}\right)$, given (i) the maximum residual time for current packet to complete transmission, (ii) a period to service $n$ PD sources $\vec{T}_{R A P}(n)$, and (iii) the time to service the first $n_{c}$ admitted CBR sources and packets of PD sources generated while servicing the first $n_{c}$ admitted CBR sources is $A_{i, 1}\left(t_{1}, t_{2}\right) \geq\left(t_{2}-t_{1}\right)-\tau_{b u s y}^{P P}-\bar{T}_{R A P}(n)-\sum_{i=1}^{n_{c}}\left(r_{c, i}\left(t_{2}-t_{1}\right)+1\right)$ $\left(\tau_{\text {busy }}^{P P}+\bar{T}_{R A P}(\bar{k})\right)$. The workload of the first VBR source generated in the period $\left(t_{1}, t_{2}\right)$ is $W_{v, 1}\left(t_{1}, t_{2}\right) \leq\left(\tau_{\text {busy }}^{P P}+\bar{T}_{R A P}(\bar{k})\right)$ $\left(r_{v, 1}\left(t_{2}-t_{1}\right)+b_{1}+1\right)$. Let $U_{v, I}(t)$ be the unfinished workload for the first VBR source at time $t$ and assume the RTT buffer is empty at time 0 , i.e. $U_{v, I}(0)=0$. Since we assume the polling token for VBR source $i$ is generated $p_{i}$ second after the connection is setup, the unfinished workload of the first VBR source at time $t$ is $U_{v, 1}(t)=W_{v, 1}(0, t)-A_{v, 1}\left(p_{1}, t\right)$. The delay for a packet of the first VBR source arrived at time $t$ is equal to the minimum time required to finish the unfinished workload at time $t$. Thus, the delay is bounded by

$$
d_{t}^{*}=\operatorname{supinf}_{t}\left\{d \geq 0: W_{v, 1}(0, t)-A_{r, 1}\left(p_{1}, t+d\right) \leq 0\right\}
$$

Since the offered load must less than the system capacity, i.e. $X_{0}+r_{v, 1}\left(\tau_{b u s y}^{P P}+\vec{T}_{R A P}(\bar{k})\right)<1$, the delay bound can be reduced to

$$
d_{1}^{*}=p_{1}+\frac{Y_{1}+Y_{0}}{1-X_{0}}
$$

In the following, we follow the same approach to further derive the delay bound for the $i$-th VBR source. Due to the preset service priority, we know that the $i$-th VBR source will receive service after all PD sources, all $n_{c}$ CBR sources and all the first $i-1$ VBR sources finished services.

First of all, the busy hour for these $n_{c}$ CBR sources is bounded by $\sum_{m=1}^{n_{\mathrm{c}}}\left(r_{c, m}\left(t_{2}-t_{1}\right)+1\right)\left(\tau_{\text {busy }}^{P P}+\bar{T}_{R A P}(\bar{k})\right)$. Then, the busy hour for these $i-1$ VBR sources can be bounded by $\left.\sum_{m=1}^{i-1}\left[\left(t_{2}+d_{m}^{*}-t_{1}\right) r_{v, m}+b_{m}+1\right)\right]\left(\tau_{\text {busy }}^{P P}+\bar{T}_{R A P}(\bar{k})\right)$. In addition, we need to further consider the busy period provided by the EOF signal resulting from polling the empty RTT buffers. This can be upper bounded by $\tau_{\text {empy }} \sum_{m=1}^{i-1}\left(\frac{t_{2}-t_{1}}{p_{m}}+1\right)$ where $\tau_{\text {empty }}$ is the time needed to transmit an EOF signal and is defined as $\tau_{\text {empty }}=\tau_{\text {poll }}+\tau_{E O F}$. Thus, the available service time for the $i$-th VBR source in $\left(t_{1}, t_{2}\right)$ is $A_{r, i} \geq\left(t_{2}-t_{1}\right)\left(1-\sum_{m=0}^{i-1} X_{m}\right)$ $-\sum_{m=0}^{i-1} Y_{m}-\sum_{m=1}^{i-1} \tau_{\text {empry }}-\sum_{m=1}^{i-1} d_{m}^{*} r_{v, m}\left(\tau_{b u s y}^{P P}+\bar{T}_{R A P}(\bar{k})\right)$.

The total number of packets for the $i$-th VBR source generated in $\left(t_{1}, t_{2}\right)$ is upper bounded by $r_{v, j}\left(t_{2}-t_{1}\right)+b_{i}+1$. Thus the workload for the $i$-th VBR source in $\left(t_{1}, t_{2}\right)$ is 
$W_{v, i}\left(t_{1}, t_{2}\right) \leq\left(r_{v, i}\left(t_{2}-t_{1}\right)+b_{i}+1\right)\left(\tau_{b u s y}^{P P}+\bar{T}_{R A P}(\bar{k})\right)=r_{v, i}\left(t_{2}-t_{1}\right)$ $\left(\tau_{b u s y}^{P P}+\bar{T}_{R A P}(\bar{k})\right)+Y_{i .} \quad$ As in Eq. (3), if $\sum_{m=0}^{n_{v}} X_{m}<1$, we can obtain the delay bound for the $i$-th VBR source

$$
d_{i}^{*}=p_{i}+\frac{\sum_{m=0}^{i} Y_{m}+\sum_{m=1}^{i-1} d_{m}^{*} r_{v, m}\left(\tau_{b u s y}^{P P}+\bar{T}_{R A P}(\bar{k})\right)+(i-1) \tau_{\text {empry }}}{1-\sum_{m=0}^{i-1} X_{m}} .
$$

\section{Admission Criterion}

Thus far, we obtain the jitter and delay constraints given $n_{c}$ CBR sources, $n_{v}$ VBR sources and $n=N-n_{c}-n_{v}$ PD sources. Based on the constraints and the preset increasing service priority, the admission criterion for the $\mathrm{CH}$ to decide if a newly generated CBR or VBR sources can be admitted are:

- A newly generated CBR source can only be admitted when the jitter bound in Theorem 1 and the delay bound in Theorem 2 are ail satisfied for every packet it generates.

- A newly generated VBR source is admitted only if the delay bound in Theorem 2 is satisfied.

\section{RESULTS AND DISCUSSIONS}

We first verify the derivation of the busy period $\bar{T}_{R A P}(n)$ in (A.1). Figure 4 compares the mean busy period with the simulation results under different traffic load and, from this figure, we conclude that (A.1) is a good approximation. We, then, simulate the proposed schemes and compared to the analytical bounds obtained in Theorem 1 and Theorem 2 . We assumed the total number of nodes within the considered cluster, $N$, are 15 and among which there are 7 CBR sources, 8 VBR sources, and 5 PD sources. The link speed is $10 \mathrm{Mbps}$, the packet size is $1 \mathrm{~K}$ bits, and the polling signal and EOF signal are all 50 bits. Therefore, we have $\tau_{\text {data }}=0.1 \mathrm{msec}, \tau_{\text {poll }}=$ $\tau_{\text {EOF }}=5 \mu \mathrm{sec}$. Furthermore, we assume $\tau_{a c k}=\tau_{\operatorname{mark}}=5 \mu \mathrm{sec}$. For simplicity, we let $\tau_{\text {data }}=1$ and normalize every parameter with respect to $\tau_{\text {data }}$, i.e., $\tau_{\text {pol }}=\tau_{E O F}=\tau_{\text {ack }}=\tau_{\text {mark }}=0.05$. We assume there are 30 random addresses, i.e. $R=30$. Besides, in order to model VBR source, we assume it is an output from an ON-OFF coder with coding speed $32 \mathrm{kbps}$ and at every instant of ON-OFF period, we assume a Bernoulli trial is used to decide the next period is $\mathrm{ON}$ with $\operatorname{Pr}(\mathrm{ON})$. We perform the simulation 10 runs and $6 \times 10^{7}$ time unit (which is equal to 600 seconds) in each run to collect the statistics. In Table 1, we find that some jitter constraints are violated and the corresponding packets are dropped. The reason is that since we reserve bandwidth for PD sources based on the mean busy period $\bar{T}_{R A P}(n)$, however, due to the random access nature of the RAP protocol, it is possible that the actual busy period $\widetilde{T}_{R A P}(n)>\widetilde{T}_{R A P}(n)$ and this will results in violation of CBR sources. In order to investigate the degree of violation, we define a CBR packet is loss if the jitter violates its jitter bound, and the packet loss rate of a CBR source is the ratio of number of loss packet to the total number of transmitted CBR packets. Figure 5 shows the packet loss rate of the CBR sources under different traffic load. In Figure 5, we find that the packet loss rate is smaller than 0.01 , which is an accept- able packet loss rate for CBR sources. From the delay tail distributions analysis in [7], we know that delays of VBR sources are uniformly distributed over the waiting period of the arrival of VBR polling tokens and are also mainly result from waiting the arrival of VBR polling token. Thus, we can improve VBR packet delay by reducing the VBR polling period $p$. However, smaller $p$ will increase the probability to poll empty VBR RTT buffer and result in wasting bandwidth and power. Since in wireless ad hoc networks, communications are achieved hop-by-hop to the intended destination. We choose $p_{i}=d_{i} / 4$ to reduce delay in a each hop. Table 2 shows the results of VBR sources.

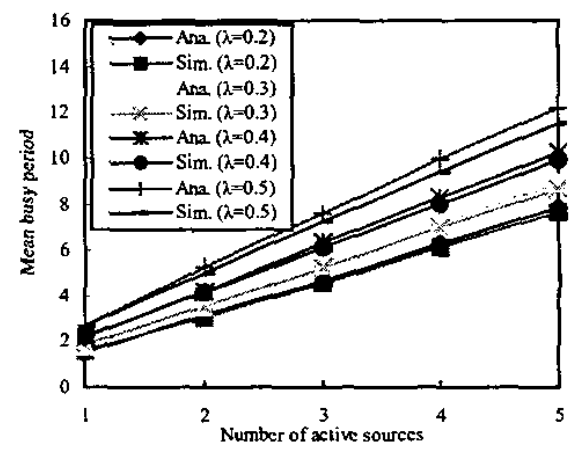

Figure 4 Mean busy period with different traffic load.

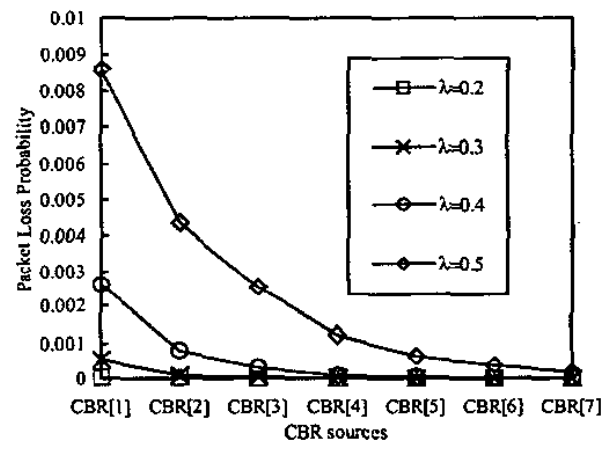

Figure 5 Packet loss rate of CBR sources with $\bar{k}=1$.

\section{CONCLUSIONS}

In this paper, we proposed a QoS guaranteed wireless access protocol, the Priority Polling with reservation (PPR) protocol which consists of Priority Polling Scheme (PPS) and Randomly Addressed Polling (RAP), to the multimedia Ad Hoc networks with Priority Data, CBR, VBR and ABR traffic sources among which the Priority Data source is considered as the highest priority to access the channel. We also provided the sufficient condition for jitter and delay constraints for CBR and VBR sources by reserving the mean busy period induced by the average number of active PD sources, $\bar{k}$, and the mean busy period induced by $n$ active PD sources. The simulation and analytical results are compared and show that through reserve bandwidth for the randomly generated PD sources, the QoS constraints for CBR and VBR sources are guaranteed with reasonable packet loss rate for CBR sources. 


\section{REFERENCES}

[1] ETSI RES, " High Performance Radio Local Area Network (HIPERLAN) Type 1, Functional Specifications," ETS $300652,1996$.

[2] J. Haartsen, "The Bluetooth Radio System," IEEE Personal Magazine, pp.28-36, Feb. 2000.

[3] K. Negus, A. Stephens, and J. Lansford, "HomeRF: Wireless Networking for the Connected Home," IEEE Personal Magazine, pp. 20-27, Feb. 2000.

[4] M. Gerla and J. T.C. Tsai, "Multicluster, Mobile, Multimedia Radio Network," ACM-Baltzar Wireless Networks, vol.1, no.3, pp.255-265, 1995.

[5] C.R. Lin and M. Gerla, "Adaptive Clustering for Mobile Wireless Networks," IEEE JSAC, vol. 15, no. 7, pp. 1265-1275, 1997.

[6] C. Zhu and M. S. Corson, "A Five-Phase Reservation Protocol (FPRP) for Mobile Ad Hoc Networks," IEEE INFOCOM'98, 1998.

[7] C.S. Chang, J.F. Chang, K.C. Chen and M.Y. You, "Guaranteed Quality-of-Service Wireless Access to ATM,"IEEE JSAC, vol. 15, no. 1, pp. 106-118, 1996.

[8] R. Ramanathan and M. Steenstrup, "Hierarchically Organized, Multihop Mobile Wireless Networks for Quality-of-Service Support," ACM-Baltzar Mobile Networks and Applications, vol. 3, pp. 101-119, 1998.

[9] K.C. Chen and C.H. Lee, "RAP-A Novel Medium Access Control Protocol for Wireless Data Networks," IEEE GLOBECOM'93, 1713-1717.

\section{APPENDIX COMPUTATION OF $\bar{T}_{R A P}(n)$}

Assume there is no collision detected. Let $\tau_{b u s y}^{R A P}=$ be the time to transmit a PD packet under the operation RAP, $\tau_{\text {busy }}^{\text {RAP }}=\tau_{\text {poll }}+\tau_{\text {data }}+\tau_{\text {ack }}$ and $\tau_{\text {over }}=\tau_{\text {poll }}+\tau_{\text {mark }}$ be the time for randomiy addressing. Let $\bar{S}_{n_{i}}$ and $\bar{M}_{n_{i}}$ be the mean number of successfully transmitted packets and the mean number of marked random number given $n_{i}$ active nodes. From the derivation in [7], we know that $\bar{S}_{n_{i}}=$ $n_{i}\left(1-\frac{1}{R}\right)^{n_{i}}$ and $\bar{M}_{n_{i}}=R\left(1-\left(1-\frac{1}{R}\right)^{n_{i}}\right)$. Then, the mean polling period to transmit $n_{i}$ active nodes is $\bar{T}\left(n_{i}\right)=\tau_{\text {over }}+$ $\bar{M}_{n_{i}} \tau_{\text {busy }}^{R A P}+\bar{T}\left(n_{i}-\bar{S}_{n_{1}}\right)$. Since $\left(n_{i}-\bar{S}_{n_{i}}\right)$ may not be an integer, for simplicity, we can approximate $\bar{T}\left(n_{i}\right)$ by

$$
\bar{T}\left(n_{i}\right)= \begin{cases}\tau_{\text {over }}+\bar{M}_{n_{i}} \tau_{\text {busy }}^{\text {RAP }}+\left(n_{i}-\bar{S}_{n_{i}}\right) \bar{T}(1) & , \text { if }\left(n_{i}-\bar{S}_{n_{i}}\right)<1 \\ \tau_{\text {over }}+\bar{M}_{n_{i}} \tau_{\text {busy }}^{\text {RAP }}+\bar{T}\left(I\left(n_{i}-\bar{S}_{n_{i}}\right)\right) & \text {, otherwise } \\ +F\left(n_{i}-\bar{S}_{n_{i}}\right) \bar{T}(1) & \end{cases}
$$

where $I(x)$ and $F(x)$ represent the integer part and fractional part of $x$. Since the probability of $k$ arrivals in a polling period of $\bar{T}\left(n_{i}\right)$ is a binomial distribution with parameters $n_{i}$ and $\left(1-e^{-\lambda_{P D} \bar{T}\left(n_{i}\right)}\right)$. The average number of PD packets that generated during $\bar{T}\left(n_{i}\right)$ is $n_{i+1}=n_{i}\left(1-e^{-\lambda_{p D} \bar{T}\left(n_{i}\right)}\right)$. Consider the case that the system switched to service PD sources with $n$ active sources initially, i.e., $n_{0}=n$ as depicted in Figure 6 . Then, the mean busy period initiated by these $n$ nodes is

$$
\bar{T}_{R A P}(n)=\sum_{n_{i}>0} \bar{T}\left(n_{i}\right)
$$

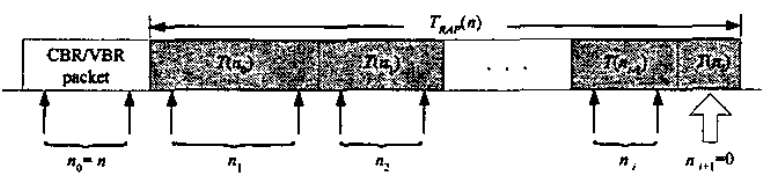

Figure 6 Busy period $\bar{T}_{R A P}(n)$ induced by initially $n_{0}=n$ active PD sources.

TABLE 1 COMPARISON BETWEEN THEOREM 1 AND SIMULATION RESULTS OF THE CBR SOURCES.

\begin{tabular}{|c|c|c|c|c|c|c|c|c|c|}
\hline \multirow{2}{*}{ CBR } & \multirow{2}{*}{$\left(r_{c, i}, j_{i}, l_{i}\right)$} & \multicolumn{2}{|c|}{$\lambda=0.2$} & \multicolumn{2}{c|}{$\lambda=0.3$} & \multicolumn{2}{|c|}{$\lambda=0.4$} & \multicolumn{2}{|c|}{$\lambda=0.5$} \\
\cline { 3 - 10 } & & Thm. 1 & Sim. & Thm. 1 & Sim. & Thm. 1 & Sim. & Thm. 1 & Sim. \\
\hline 1 & $(0.040,50,0.01)$ & 8.852 & 13.950 & 9.947 & 16.700 & 11.291 & 22.400 & 13.199 & 27.850 \\
\hline 2 & $(0.033,60,0.01)$ & 12.666 & 14.797 & 14.381 & 18.303 & 16.579 & 27.003 & 19.816 & 32.397 \\
\hline 3 & $(0.024,70,0.01)$ & 17.069 & 15.417 & 19.606 & 22.433 & 22.985 & 28.167 & 28.172 & 40.567 \\
\hline 4 & $(0.020,80,0.01)$ & 21.798 & 17.100 & 25.300 & 25.200 & 30.119 & 32.050 & 37.808 & 46.250 \\
\hline 5 & $(0.015,100,0.01)$ & 26.962 & 19.683 & 31.622 & 25.717 & 38.233 & 35.417 & 49.217 & 48.717 \\
\hline 6 & $(0.012,120,0.01)$ & 32.301 & 17.833 & 38.226 & 26.683 & 46.855 & 38.683 & 61.729 & 52.183 \\
\hline 7 & $(0.01,150,0.01)$ & 37.827 & 19.650 & 45.132 & 24.100 & 56.017 & 40.350 & 75.443 & 57.900 \\
\hline
\end{tabular}

TABLE 2 COMPARISON BETWEEN THEOREM 2 AND SIMULATION RESULTS OF THE VBR SOURCES.

\begin{tabular}{|c|c|c|c|c|c|c|c|c|c|c|}
\hline VBR & \multirow{2}{*}{$\operatorname{Pr}(\mathrm{ON})$} & \multirow{2}{*}{$\left(r_{v i}, b_{i}, d_{i}\right)$} & \multicolumn{2}{|c|}{$\lambda=0.2$} & \multicolumn{2}{c|}{$\lambda=0.3$} & \multicolumn{2}{|c|}{$\lambda=0.4$} & \multicolumn{2}{c|}{$\lambda=0.5$} \\
\cline { 5 - 10 } & & & Thm. 2 & Sim. & Thm. 2 & Sim. & Thm. 2 & Sim. & Thm. 2 & Sim. \\
\hline 1 & 0.046 & $\left(1.48 \times 10^{-3}, 10,1000\right)$ & 339.175 & 259.110 & 357.005 & 267.323 & 384.275 & 270.197 & 434.518 & 290.143 \\
\hline 2 & 0.045 & $\left(1.42 \times 10^{-3}, 10.1000\right)$ & 387.988 & 257.524 & 415.805 & 266.671 & 458.638 & 277.359 & 538.237 & 276.844 \\
\hline 3 & 0.043 & $\left(1.36 \times 10^{-3}, 8,1000\right)$ & 429.225 & 258.272 & 465.715 & 264.284 & 522.225 & 268.994 & 628.137 & 294.225 \\
\hline 4 & 0.041 & $\left(1.31 \times 10^{-3}, 8,1000\right)$ & 471.094 & 258.510 & 516.556 & 260.312 & 587.322 & 270.615 & 721.033 & 283.687 \\
\hline 5 & 0.040 & $\left(1.27 \times 10^{-3}, 8,1000\right)$ & 513.591 & 258.658 & 568.321 & 263.263 & 653.925 & 275.641 & 816.949 & 294.559 \\
\hline 6 & 0.037 & $\left(1.18 \times 10^{-3}, 5,1000\right)$ & 543.870 & 258.196 & 258.196 & 262.227 & 702.450 & 276.537 & 888.570 & 275.963 \\
\hline 7 & 0.033 & $\left(1.09 \times 10^{-3}, 5,1000\right)$ & 574.350 & 261.395 & 643.062 & 264.421 & 751.560 & 272.986 & 961.526 & 285.189 \\
\hline 8 & 0.032 & $\left(1.00 \times 10^{-3}, 5,1000\right)$ & 604.977 & 258.641 & 680.840 & 269.653 & 801.128 & 265.386 & 1035.574 & 302.325 \\
\hline
\end{tabular}

